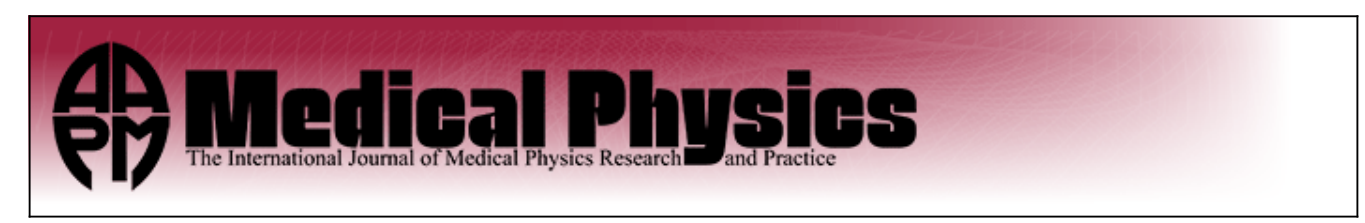

\title{
Optimization of oncological 18F-FDG PET/CT imaging based on a multiparameter analysis
}

Vinicius O. Menezes, Marcos A. D. Machado, Cleiton C. Queiroz, Susana O. Souza, Francesco d'Errico, Mauro Namías, Ticiana F. Larocca, and Milena B. P. Soares

Citation: Medical Physics 43, 930 (2016); doi: 10.1118/1.4940354

View online: http://dx.doi.org/10.1118/1.4940354

View Table of Contents: http://scitation.aip.org/content/aapm/journal/medphys/43/2?ver=pdfcov

Published by the American Association of Physicists in Medicine

\section{Articles you may be interested in}

Local respiratory motion correction for PET/CT imaging: Application to lung cancer

Med. Phys. 42, 5903 (2015); 10.1118/1.4930251

Evaluation of respiratory and cardiac motion correction schemes in dual gated PET/CT cardiac imaging Med. Phys. 41, 072504 (2014); 10.1118/1.4881099

PET/CT imaging evidence of FUS-mediated (18)F-FDG uptake changes in rat brain

Med. Phys. 40, 033501 (2013); 10.1118/1.4789916

Quantitative accuracy of PET/CT for image-based kinetic analysis

Med. Phys. 35, 3086 (2008); 10.1118/1.2937439

Improved quantitation for PET/CT image reconstruction with system modeling and anatomical priors

Med. Phys. 33, 4095 (2006); 10.1118/1.2358198

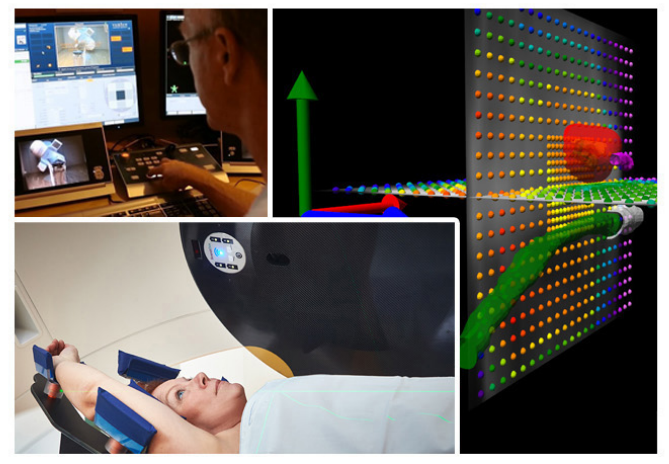

ScandiDos Delta 4 family

offers precise and easy

QA from plan to the last

fraction

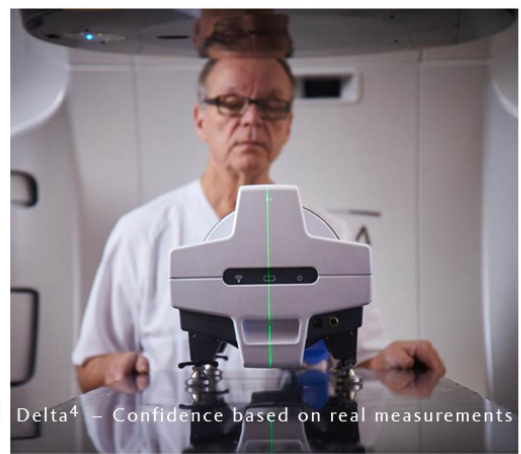




\title{
Optimization of oncological ${ }^{18} \mathrm{~F}$-FDG PET/CT imaging based on a multiparameter analysis
}

\author{
Vinicius O. Menezes ${ }^{a)}$ \\ Nuclear Medicine Department, São Rafael Hospital, Salvador 41720-375, Brazil and Nuclear Medicine \\ Department, Hospital das Clínicas da Universidade Federal de Pernambuco/Ebserh, Recife 50670-901, Brazil
}

Marcos A. D. Machado

Nuclear Medicine Department, São Rafael Hospital, Salvador 41720-375, Brazil and Nuclear Medicine Department, Hospital das Clínicas da Universidade Federal de Bahia/Ebserh, Salvador 40110-060, Brazil

Cleiton C. Queiroz

Nuclear Medicine Department, São Rafael Hospital, Salvador 41720-375, Brazil and Nuclear Medicine Department, Hospital Universitário Professor Alberto Antunes/Ebserh, Maceió 57072-900, Brazil

Susana O. Souza

Department of Physics, Universidade Federal de Sergipe, São Cristóvão 49100-000, Brazil

Francesco d'Errico

Department of Diagnostic Radiology, Yale University School of Medicine, New Haven, Connecticut 06520 and School of Engineering, University of Pisa, Pisa 56126, Italy

Mauro Namías

Fundación Centro Diagnóstico Nuclear, Buenos Aires C1417CVE, Argentina

Ticiana F. Larocca

Centro de Biotecnologia e Terapia Celular, São Rafael Hospital, Salvador 41253-190, Brazil

Milena B. P. Soares

Centro de Biotecnologia e Terapia Celular, São Rafael Hospital, Salvador 41253-190, Brazil and Fundação Oswaldo Cruz, Centro de Pesq. Gonçalo Moniz, Salvador 40296-710, Brazil

(Received 22 August 2015; revised 29 November 2015; accepted for publication 10 January 2016; published 26 January 2016)

Purpose: This paper describes a method to achieve consistent clinical image quality in ${ }^{18}$ F-FDG scans accounting for patient habitus, dose regimen, image acquisition, and processing techniques.

Methods: Oncological PET/CT scan data for 58 subjects were evaluated retrospectively to derive analytical curves that predict image quality. Patient noise equivalent count rate and coefficient of variation $(\mathrm{CV})$ were used as metrics in their analysis. Optimized acquisition protocols were identified and prospectively applied to 179 subjects.

Results: The adoption of different schemes for three body mass ranges ( $<60 \mathrm{~kg}, 60-90 \mathrm{~kg},>90 \mathrm{~kg}$ ) allows improved image quality with both point spread function and ordered-subsets expectation maximization-3D reconstruction methods. The application of this methodology showed that CV improved significantly $(p<0.0001)$ in clinical practice.

Conclusions: Consistent oncological PET/CT image quality on a high-performance scanner was achieved from an analysis of the relations existing between dose regimen, patient habitus, acquisition, and processing techniques. The proposed methodology may be used by PET/CT centers to develop protocols to standardize PET/CT imaging procedures and achieve better patient management and cost-effective operations. (C) 2016 American Association of Physicists in Medicine. [http://dx.doi.org/10.1118/1.4940354]

Key words: PET/CT, optimization, image quality consistency, low dose, ${ }^{18} \mathrm{~F}-\mathrm{FDG}$

\section{INTRODUCTION}

Oncological ${ }^{18} \mathrm{~F}$-FDG PET/CT is recognized as a powerful diagnostic and staging technique for many types of tumors. ${ }^{1-4}$ Optimal PET/CT application should result in consistent diagnostic image quality with minimal radiological risks and adequate patient comfort. Despite efforts to develop standard PET/CT imaging protocols, ${ }^{5-10}$ image quality degradation is often reported, mainly in obese subjects. ${ }^{11-15}$
Because image quality is heavily influenced by patient size, PET centers should strive for patient-specific imaging protocols to assure consistent clinical evaluations. ${ }^{11,12,14}$ This work provides optimal patient-specific protocols to match clinical resources of injected activity and acquisition durations.

Several studies have proposed ${ }^{18} \mathrm{~F}$-FDG linear or quadratic injected dose regimens aiming at consistent image quality and accounting for patient body mass, body mass index 
(BMI), image acquisition, and noise equivalent counts (NECs). ${ }^{12,14,16-19}$ Ideally, PET should be performed with optimal acquisition parameters to achieve homogeneous image quality over a specific population. Additionally, advanced image reconstruction using methods such as point spread function (PSF) modeling has shown to improve the image quality and imaging protocols. ${ }^{13,18}$ The growing availability of high-performance equipment, reconstruction software, and quantitative methods provides new opportunities for improved imaging and patient management ${ }^{7,13,16-20}$ motivating our efforts to evaluate protocol selections on modern systems. Image noise and data of NEC are dependent on scanner, activity concentration, and patient habitus. ${ }^{12}$ de Groot et al. performed an analysis of image noise across a range of patients leading to a suggested quadratic injected-dosing scheme. ${ }^{18}$ Other authors have performed similar noise analysis on raw data evaluating NEC to show the relationship of NEC with different patientspecific parameters. ${ }^{12,19}$

Our current work provides similar insight as these prior protocol development efforts, relating NEC and image noise to patient parameters, and provides the new, additional value of recommending patient-specific protocols for both injected activity and acquisition duration. These protocols would be valuable for a variety of clinical settings including those receiving a bulk dose each day and needing to distribute the dose in an optimal manner across patients, or for clinics that inject identical activities and need to vary the acquisition durations to achieve similar image quality.

This study investigated patient-dependent parameters (PDPs), dose regimen, and data acquisition techniques, aiming at defining a method for high and consistent image quality of low-dose PET/CT scans. Concurrently, the effects of PSF reconstruction algorithms were evaluated. Overall, we propose a methodology to achieve standardized noise levels based on the optimization of acquisition parameters (activity concentration and acquisition time). This work provides a feasible methodology that might be applied in PET centers to achieve uniform image quality over patient population for different scanners and processing techniques. By using this method, PET studies might be performed according to a chosen ${ }^{18} \mathrm{~F}$ FDG administered activity and the acquisition time will be analytically defined.

\section{MATERIALS AND METHODS}

\section{A. Patient population}

A total of $85{ }^{18}$ F-FDG-PET adult studies were gathered over a 3-month period and retrospectively evaluated. Exclusion criteria were pregnancy or nursing, motor difficulties, liver metastases, hyperglycemia ${ }^{7}$ at the time of tracer administration, or a delay exceeding $90 \mathrm{~min}$ between ${ }^{18} \mathrm{~F}-\mathrm{FDG}$ injection and image acquisition. The study was approved by the Monte Tabor-Hospital São Rafael ethical board (ID: 27558714.4.0000.0048), submitted to the Brazilian clinical studies platform (UTN U1111-1163-0663) and to the NIH Clinical Trials (NCT02378337). Written informed consent was obtained from all subjects.

\section{B. PET/CT imaging}

PET/CT imaging was performed on a LSO-based PET Siemens Biograph TruePoint TrueV (Knoxville, TN, USA) combined with a 16-slice helical CT scanner (Emotion 16; Siemens). The PET component operates fully in 3D mode and incorporates four detector rings of 48 detector blocks, each comprising $13 \times 13$ crystals $\left(4 \times 4 \times 20 \mathrm{~mm}^{3}\right)$ coupled to four photomultiplier tubes. This configuration covers an axial field-of-view (FOV) of $216 \mathrm{~mm}$ and a transaxial FOV of $700 \mathrm{~mm}$ diameter. The wide detector ring of the system allows a high-performance, ${ }^{20}$ as confirmed by in house measurements $^{21}$ [sensitivity $=8.1 \mathrm{cps} / \mathrm{kBq}$ at FOV center and noise equivalent count rate $(\mathrm{NECR})=179 \mathrm{kcps}$ at $33 \mathrm{kBq} / \mathrm{ml}]$.

$\mathrm{PET} / \mathrm{CT}$ was performed according to the clinical protocol of Hospital São Rafael for tumor PET imaging. Subjects fasted for $6 \mathrm{~h}$ prior to the ${ }^{18} \mathrm{~F}-\mathrm{FDG}$ injection. The mean ${ }^{18} \mathrm{~F}-\mathrm{FDG}$ injection dose was $3.44 \pm 0.58 \mathrm{MBq} / \mathrm{kg}$ (range, 1.81-4.63 MBq/kg). PET scans started $73 \pm 15 \mathrm{~min}$ (range, 50-90 $\mathrm{min}$ ) after injection. Images were acquired from the midthigh to the vertex of the skull of subjects in supine position with the arms positioned above the head. CT scans were done during shallow breathing using the Siemens CareDose4D dose modulation at $110 \mathrm{kVp}(n=56)$ and $130 \mathrm{kVp}(n=2), 0.6 \mathrm{~s}$ rotation time, $5 \mathrm{~mm}$ slice thickness, and $9.6 \mathrm{~mm}$ collimation; a standard soft tissue reconstruction kernel (Siemens B30s) was used. Before the whole body PET scan, an image of the liver was acquired in list-mode (one bed position) during $360 \mathrm{~s}$ and the previous CT scan was used for attenuation correction. Images from the list-mode data were reconstructed at incremental $30 \mathrm{~s}$ intervals up to $360 \mathrm{~s}$ (12 images per patient) with a $168 \times 168$ matrix size $\left(4.07 \times 4.07 \times 2.00 \mathrm{~mm}^{3}\right.$ voxels $)$. Iterative reconstruction algorithms previously validated in our clinical practice ${ }^{22}$ were used: (1) ordered-subsets expectation maximization (OSEM3D) with 3 iterations, 21 subsets, and a $5 \mathrm{~mm}$ Gaussian filter and (2) point spread function ordinary Poisson (PSF) with 2 iterations, 21 subsets, and a $2 \mathrm{~mm}$ Gaussian filter. Data were corrected for random coincidences, normalization, dead time losses, scatter, and attenuation.

\section{C. Image analysis}

All images were analyzed with Syngo.VIA version VA30 (Siemens Healthcare). Data and image quality analyses were done measuring the NECR and the coefficient of variation (CV) in the liver, which presents a uniform ${ }^{18} \mathrm{~F}-\mathrm{FDG}$ uptake in normal subjects. ${ }^{7,18,22}$

The NECR is a raw data quality metric, not accounting for normalization, attenuation correction, spatial resolution effects, and reconstruction algorithms. The NECR was calculated as

$$
\mathrm{NECR}=\frac{T^{2}}{T+S+k R},
$$

where $T$ is the true coincidence count rate, $S$ is the scatter coincidence count rate, $R$ is the random coincidence count rate, and $k=1$ (smoothed random correction). ${ }^{23}$ The true and random coincidence count rates were estimated by the scanner and obtained from the full sinogram data header. The scatter 
coincidence rate was calculated as

$$
S=s f \cdot P,
$$

where $s f$ is the scatter fraction estimated by the scanner and obtained from reconstructed images and $P$ is the total prompt event rate.

The quality of the 696 clinical PET image acquisitions (12 images $\times 58$ subjects) was analyzed using the $\mathrm{CV}$ in the liver as a noise metric. We defined a spherical volume of interest of $3 \mathrm{~cm}$ diameter in the largest liver section to compute the mean and standard deviation (SD) counts. ${ }^{24-26}$ The CV in the liver was calculated as

$$
\mathrm{CV}=\frac{\mathrm{SD}}{\text { Mean }}
$$

Since positron emission is a random process following Poisson statistics, we can derive a first-order approximation for the $\mathrm{CV}$ from the total collected counts as

$$
\mathrm{CV}=\frac{\mathrm{SD}}{\text { Mean }} \sim \frac{\sqrt{N}}{N}=\frac{1}{\sqrt{N}} \sim \frac{1}{\sqrt{A \cdot t}},
$$

where $N$ is the number of measured disintegrations, $A$ is the activity $(\mathrm{MBq})$ in the volume of interest, and $t$ is the scan time per bed position (T.BED). In clinical imaging, $\mathrm{CV}$ is a function of equipment performance, injected dose, acquisition time, reconstruction, processing, and PDP. ${ }^{18} \mathrm{~F}-\mathrm{FDG}$ uptake and NECR are usually related to patient body mass, but other PDP may be more adequate. ${ }^{18,25}$ For this reason, we evaluated the influence of PDPs on NECR and CV accounting for patient habitus: body mass, BMI, ${ }^{27}$ lean body mass (LBM) as defined by Hume, ${ }^{28}$ body surface area (BSA), ${ }^{29}$ and effective diameter (ED). ${ }^{30,31}$ Once Eq. (4) is defined by a power function, we sought to find the coefficients in Eq. (5) that explain our scanner, reconstruction, and population behavior, ${ }^{17,18}$

$$
\mathrm{CV}=a *\left(\mathrm{~T} . \mathrm{BED} * \frac{A(\mathrm{acq})}{\mathrm{PDP}}\right)^{b},
$$

where $a$ and $b$ are the fitting coefficients and $A(\mathrm{acq})$ is the activity at imaging time (injected activity corrected for physical decay).

In this paper, NEC was used as an intermediate step to determine the quality of the raw data acquired, whereas $\mathrm{CV}$ was used as a measure of image noise. The statistics in the raw data follow the Poisson distribution whereas different reconstruction algorithms and reconstruction parameters can lead to different relationships between NEC and CV. NEC depends on scanner characteristics, activity concentration and patient habitus (body mass, LBM, BSA, BMI, ED). Thus, we used NEC to determine the best dose regimen for our population.

In order to account for subjective assessment of image quality, different ranges of noise levels in the liver of 18 subjects (five images of each patient reconstructed with OSEM 3D and PSF) were randomly analyzed by three independent blinded reviewers. The images were graded by count density and uniformity, edge definition, and noise by using a 5-point scale: 1 (poor) to 5 (excellent).

\section{D. Protocol validation}

To verify the application of methodology in clinical routine, we conducted a second phase of the study prospectively in 250 subjects (phase 2) using inclusion and exclusion criteria of the retrospective study (phase 1). Acquisition time per bed position was analytically defined according to Eq. (5). The image noise in the liver region was analyzed using the same technique as phase 1 .

\section{E. Statistical analysis}

In our statistical data analysis, we used GraphPad Prism 5.01 (GraphPad Software, Inc., La Jolla, CA, USA). Determination coefficients $\left(R^{2}\right)$ were applied to determine the PDP that better matched the power fit with a $95 \%$ confidence interval. The residuals of the robust fit were analyzed to identify outliers (Rout method) with a coefficient $Q$ equal to $1 \%$. The $F$ test was applied to identify differences between the power fits, the unpaired $t$ test was applied to identify differences between phase 1 and 2 for subject characteristics, $\mathrm{CV}$, and dose regimen values, and $p<0.05$ was considered to be statistically significant. We also performed multivariate analysis to assess independent variable predictors of $\mathrm{CV}$ in Eq. (5), including in our model the following variables: body mass, LBM, BMI, BSA, and ED. Graphs were generated with GraphPad Prism 5.01, OriginPro 9.0.0 (OriginLab Corporation, Northampton, MA, USA) and SigmaPlot 12 [Systat Software, Inc. (SSI), San Jose, CA, USA].

\section{RESULTS}

\section{A. Study population}

\begin{tabular}{|c|c|c|c|}
\hline Patient-dependent parameter & PHASE $1(n=58)$ & PHASE $2(n=179)$ & $\begin{array}{l}p \text { value } \\
\text { of } t \text { test }\end{array}$ \\
\hline Age (yr) & $56.3 \pm 16.5($ range, $18-88)$ & $54.4 \pm 16.9($ range, $18-93)$ & - \\
\hline Body mass (kg) & $71.12 \pm 15.59($ range, $39-120)$ & $72.84 \pm 16.35($ range, $43-120)$ & 0.43 \\
\hline Height $(\mathrm{cm})$ & $163 \pm 10($ range, $140-180)$ & $165 \pm 9($ range, $144-193)$ & 0.75 \\
\hline Body mass index $\left(\mathrm{kg} \mathrm{m}^{-2}\right)$ & $26.70 \pm 5.52($ range, $15.6-40.2)$ & $26.16 \pm 5.15($ range, $15.82-42.98)$ & 0.96 \\
\hline Lean body mass (Hume, kg) & $46.96 \pm 8.30($ range, $31.2-69.9)$ & $47.80 \pm 8.40($ range, $32.12-71.3)$ & 0.85 \\
\hline Body surface area (Du Bois, $\mathrm{m}^{2}$ ) & $1.76 \pm 0.21$ (range, 1.3-2.3) & $1.77 \pm 0.22($ range, $1.2-2.3)$ & 0.79 \\
\hline Effective diameter (Brady, cm) & $27 \pm 3($ range, $20-35)$ & $26 \pm 4$ (range, $19-34)$ & 0.88 \\
\hline
\end{tabular}

The study population of phase 1 was 58 subjects $(64 \%$ females) after the exclusion criteria. The mean body mass was

TABLE I. Study population.

Note: Values are expressed as mean \pm standard deviation (range, minimum-maximum). 

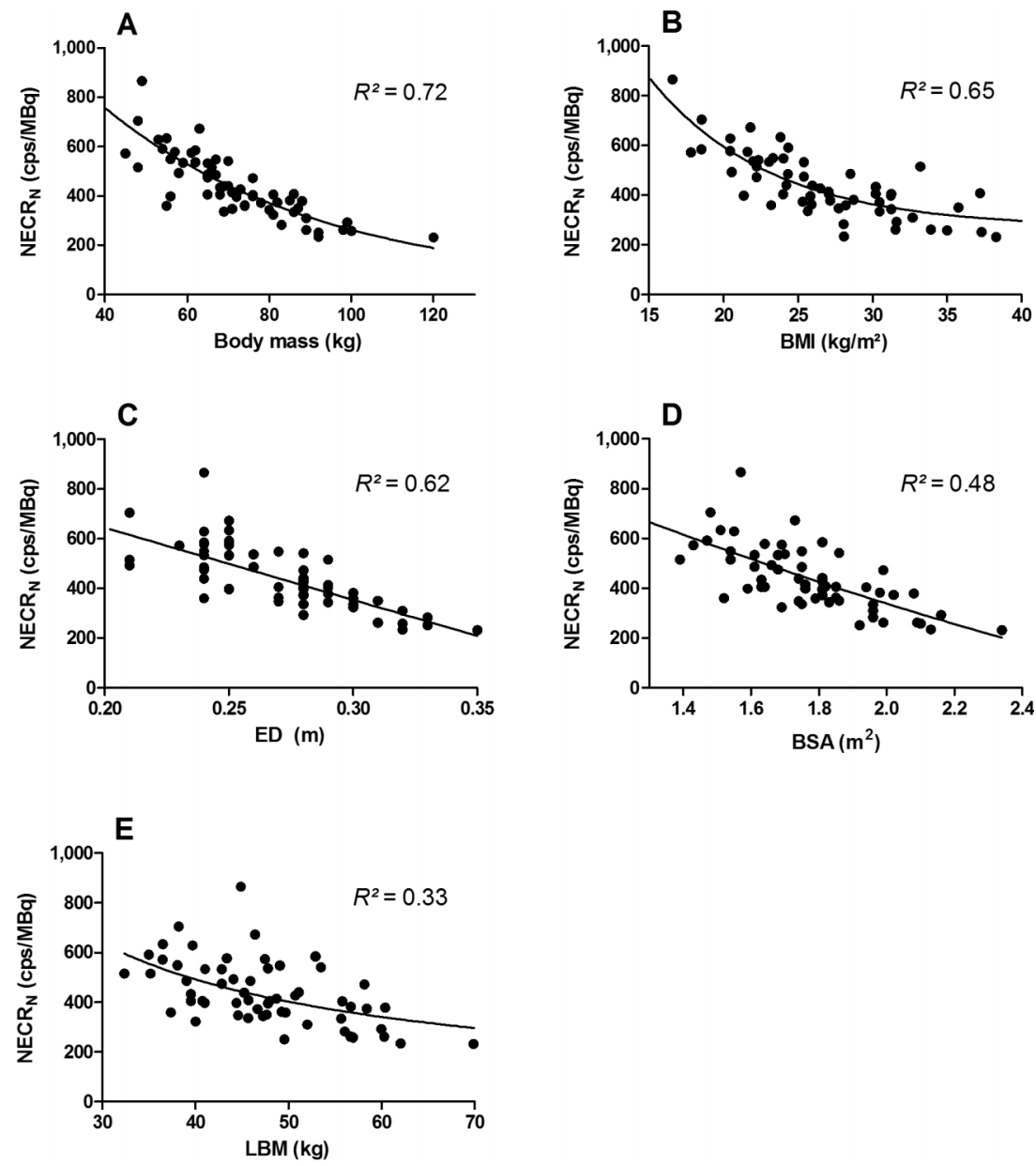

FIG. 1. Data points of 58 subjects of $\mathrm{NECR}_{N}$ vs body mass (A), BMI (B), effective diameter (C), BSA (D), and LBM (E). The lines in the graphs are the result of exponential regression of the data.

$71.12 \pm 15.59 \mathrm{~kg}$ (range, $39-120 \mathrm{~kg}$ ) and the mean height was $163 \pm 10 \mathrm{~cm}$ (range, $140-180 \mathrm{~cm}$ ). Three body mass groups were defined as the following: below $60 \mathrm{~kg}(n=16), 60-90 \mathrm{~kg}$ $(n=36)$, and over $90 \mathrm{~kg}(n=6)$. An overview of subject characteristics is given in Table I.

\section{B. Noise equivalent count rate}

To account for PDP in data analysis, we normalized the NECR by the ${ }^{18}$ F-FDG radioactivity at the time of image acquisition $\left(\mathrm{NECR}_{N}\right)$ and fitted exponential curves of $\mathrm{NECR}_{N}$ versus PDP (Fig. 1). Other fits were tested but had weaker relations.

\section{C. Image noise}

For both reconstruction methods, OSEM3D and PSF (Table II), power fits of CV versus [T.BED*A(acq)/PDP], i.e., count density normalized for PDPs, were done.

In our sample, body mass was the PDP with the strongest relations $\left(R^{2}=0.82\right.$ in OSEM3D, $p<0.001 ; R^{2}=0.86$ in PSF, $p<0.001)$.

TABLE II. Fit for CV with various patient-dependent parameters.

\begin{tabular}{|c|c|c|c|c|c|c|c|c|}
\hline \multirow[b]{2}{*}{ Patient-dependent parameter } & \multicolumn{4}{|c|}{ CV (OSEM3D) } & \multicolumn{4}{|c|}{ CV (PSF) } \\
\hline & $a$ & $b$ & $R^{2}$ & $p$ value of $F$ test & $a$ & $b$ & $R^{2}$ & $p$ value of $F$ test \\
\hline Body mass $\left(\mathrm{kg}^{-1}\right)$ & 1.18 & -0.38 & 0.82 & - & 1.20 & -0.40 & 0.86 & - \\
\hline Body mass index $\left(\mathrm{kg} \mathrm{m}^{-2}\right)$ & 1.60 & -0.37 & 0.77 & $<0.001$ & 1.89 & -0.41 & 0.82 & $<0.001$ \\
\hline Lean body mass (Hume, $\mathrm{kg}$ ) & 6.14 & -0.34 & 0.69 & $<0.001$ & 1.26 & -0.34 & 0.58 & $<0.001$ \\
\hline Body surface area (du Bois, $\mathrm{m}^{2}$ ) & 1.32 & -0.38 & 0.77 & $<0.001$ & 1.48 & -0.41 & 0.80 & $<0.001$ \\
\hline Effective diameter (Brady, m) & 4.42 & -0.37 & 0.76 & $<0.001$ & 5.83 & -0.41 & 0.81 & $<0.001$ \\
\hline
\end{tabular}

Note: Data were fitted with a power adjustment. $a$ and $b$ are fitting parameters [Eq. (5)]. $R^{2}$ : coefficient of determination. CV: coefficient of variation. OSEM3D: ordered-subsets expectation maximization. PSF: point spread function ordinary Poisson. 
TABLE III. Results of multiple linear regression analysis.

\begin{tabular}{lcc}
\hline \hline Patient-dependent parameter & $\begin{array}{c}\text { Standardized regression } \\
\text { coefficients } \beta\end{array}$ & $\begin{array}{c}\text { Probability } \\
p\end{array}$ \\
\hline Body mass $\left(\mathrm{kg}^{-1}\right)$ & -1.221 & 0.007 \\
Body mass index $\left(\mathrm{kg} \mathrm{m}^{-2}\right)$ & -0.866 & 0.059 \\
Lean body mass $(\mathrm{Hume}, \mathrm{kg})$ & 0.046 & 0.896 \\
Body surface area (Du Bois, $\left.\mathrm{m}^{2}\right)$ & 1.944 & 0.004 \\
Effective diameter (Brady, $\mathrm{m})$ & -0.672 & 0.105 \\
\hline \hline
\end{tabular}

The results showed that only body mass and BSA remained as significant predictors of $\mathrm{CV}$, after controlling for the other variables (Table III).

CV distributions derived from all 696 images are shown in Fig. 2 and indicate a strong relation between count density and image noise within the body mass range.

Figure 3 illustrates PET image quality degradation with increasing patient body mass: heavier subjects tend to require more counts to achieve a given noise level.

This may be addressed by increasing dose concentration or/and acquisition time. Analysis of Fig. 3 suggests a better $\mathrm{CV}$ distribution for each body mass group. For this reason, the body mass range was divided within three groups (subjects below $60 \mathrm{~kg}$, between 60 and $90 \mathrm{~kg}$ and over $90 \mathrm{~kg}$ ).

In order to determine optimal total counts for three body mass ranges of the subjects, we plotted the coefficient of variation $\mathrm{CV}$ as a function of T.BED*A(acq) for OSEM3D and for PSF reconstructions (Fig. 4).

We chose these body mass ranges because we observed an increase in CV data relation for subjects below $60 \mathrm{~kg}$, subjects between 60 and $90 \mathrm{~kg}$, and for subjects over $90 \mathrm{~kg}$ (Table IV); these relations present $p<0.05$, except for the ranges $<60 \mathrm{~kg}$ and 60-90 kg in PSF reconstruction, when $p<0.3847$.

Figure 5 shows the results of image quality by subjective assessment reconstructed with (A) OSEM3D and (B) PSF.

The 90 images correspond to six subjects categorized in each body mass range reconstructed with [(A)-(E)] OSEM3D and $[(\mathrm{F})-(\mathrm{J})]$ PSF. The subject images were distributed from 9\% to $21 \%$ noise level (Fig. 6).

Table $\mathrm{V}$ shows different dose regimen and acquisition parameters adjustments for $12 \%$ coefficient of variation. By using the proposed methodology, the duration of acquisition might be adjusted accordingly to analytically achieve [Eq. (5)]

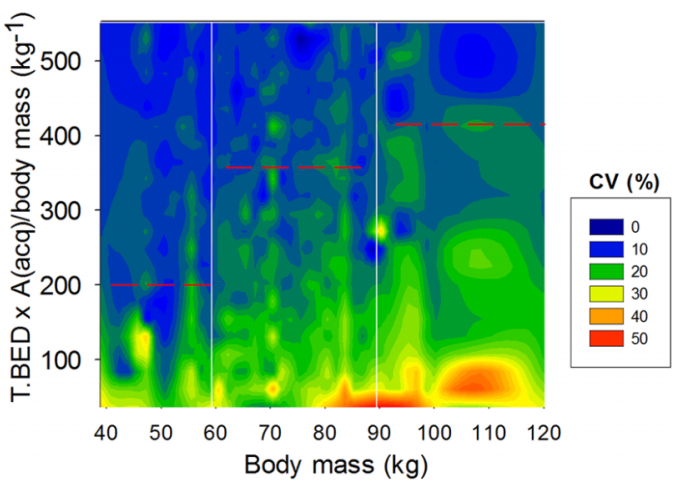

FIG. 3. Coefficient of variation (CV) 3D representation of 696 images vs count density ( $y$-axis) vs body mass ( $x$-axis) for OSEM3D reconstruction. $\mathrm{CV}$ is represented as a color map of six color groups. White lines represent the segmentation of body mass (below $60 \mathrm{~kg}$, subjects between 60 and $90 \mathrm{~kg}$, and for subjects over $90 \mathrm{~kg}$ ). Dashed lines indicate the values considered as acceptable of CV for each group (visual selection of CV between $10 \%$ and 20\%). OSEM3D: ordered-subsets expectation maximization.

the required coefficient of variation based on the injected activity concentration.

\section{D. Protocol validation}

Since visual perception was indexed by multiple coefficient of variation in phase 1 , we defined an excellent image quality perception translated into $12 \%$ coefficient of variation to be the standard of quality over the prospective studies.

The population of phase 2 consisted of 179 subjects (54\% females). The mean body mass was $72.8 \pm 16.4 \mathrm{~kg}$ (range, $43-120 \mathrm{~kg}$ ) and the mean height was $165.1 \pm 9.2 \mathrm{~cm}$ (range, 144-193 cm). Table I shows there are no differences between population characteristics of phase 1 and 2 . The mean ${ }^{18} \mathrm{~F}-\mathrm{FDG}$ injection dose was $3.29 \pm 0.78 \mathrm{MBq} / \mathrm{kg}$ (range, 2.22-6.25 MBq $/ \mathrm{kg}$ ) (there is no difference between dose regimen of phase 1 and 2, $p=0.1672$ ) and PET scans started $72 \pm 12 \mathrm{~min}$ (range, $50-90 \mathrm{~min}$ ) (there is no difference between dose regimen of phase 1 and $2, p=0.9890)$ after injection. The CT parameters were at $110 \mathrm{kVp}(n=175)$ and $130 \mathrm{kVp}$ $(n=4)$, and PET images were reconstructed with OSEM3D as previously described in the first phase. Figure 7 represents the comparison of $\mathrm{CV}$ between phase 1 and 2 for the whole body acquisition, showing a significant improvement in image noise $(p<0.0001)$.
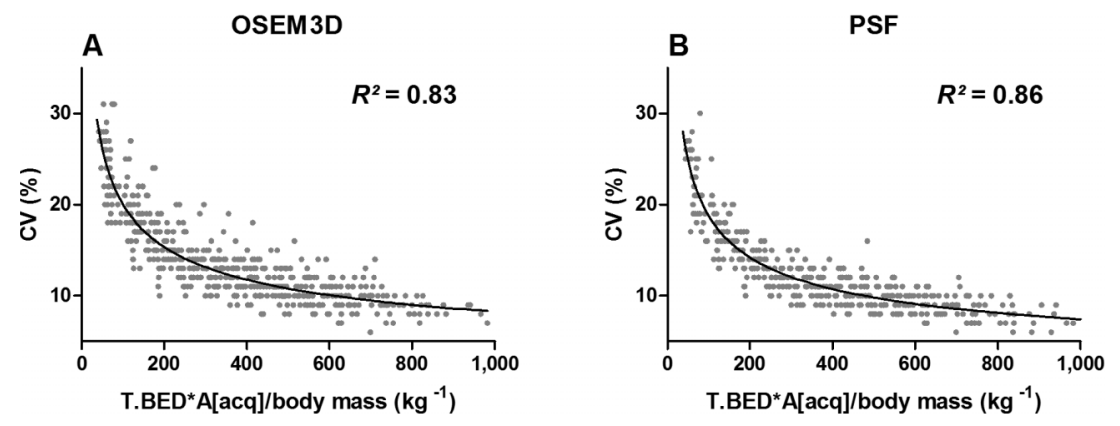

FIG. 2. Data points of 696 images and power fits of noise in the liver (CV) vs T.BED*A(acq)/body mass for OSEM3D (A) and PSF (B) reconstruction. The lines in the graphs are the result of power regression of the data. 


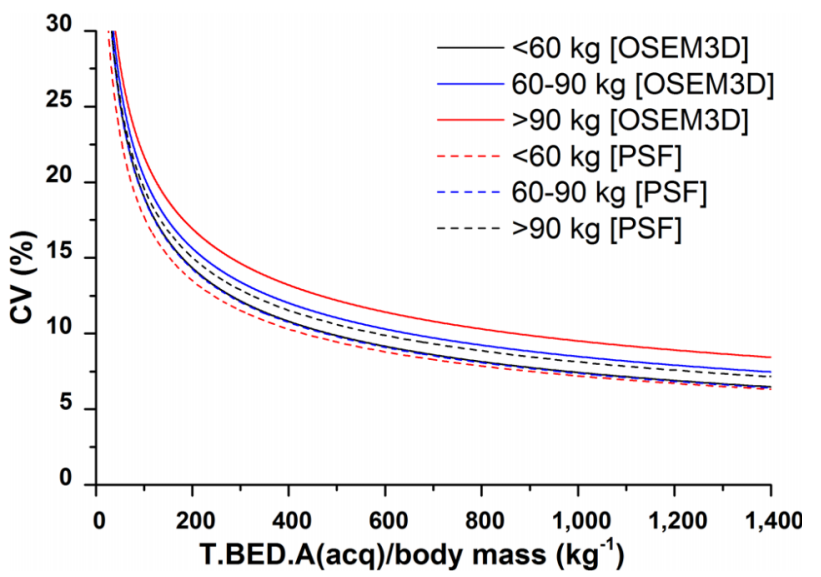

FIG. 4. The lines in the graphs represent the power regression of the data for 696 images. Power fits of coefficient of variation (CV) in the liver vs T.BED*A(acq)/body mass [Eq. (5)] for OSEM3D (solid line) and PSF (dashed line) reconstructions. The body mass fit ranges are $<60 \mathrm{~kg}(n=16)$, $60-90 \mathrm{~kg}(n=36),>90 \mathrm{~kg}(n=6)$. OSEM3D: ordered-subsets expectation maximization. PSF: point spread function ordinary Poisson.

\section{DISCUSSION}

NECR is a complex function of dose concentration and patient habitus. ${ }^{12,19,32}$ Our findings suggest that imaging heavier subjects poses two important issues: (i) increasing the activity may not improve NECR significantly and (ii) $\mathrm{NECR}_{N}$ decreases exponentially as body mass increases. Thus, dedicated protocols must be identified and applied for heavier subjects in order to minimize radiation hazards and produce adequate and consistent image quality. Besides the parameters described for NECR, the image noise observed in reconstructed images also depends strongly on image acquisition and processing parameters.

Prior studies have suggested various dose regimens, including linear ${ }^{5-7,10}$ and quadratic regimens based on PDPs (Ref. 18) as well as fixed dose regimens for all subjects. The linear dose regimen applied in our study results in a degradation of image quality with increasing body mass. A quadratic dose regimen can address this problem, but patient doses become high. Thus, we sought alternative protocols for consistent quality imaging of the examined population.

Our findings demonstrate that body mass is the PDP, most suitable for our protocol, and that $\mathrm{NECR}_{N}$ best relates with patient body mass $\left(R^{2}=0.72\right)$. Although our study examined a limited population of 58 subjects, we were able to derive 696 images to track data of different noise levels and count density within a patient range of body mass. Some earlier studies focused on the activity at injection time..$^{7,12,13,16,33}$ However, the count density derives from the activity at imaging time. Therefore, normalizing to the activity at imaging time diminishes the bias due to F-18 physical decay, most affected by delays due to patient management logistics. Effective half-life would be more realistic, but it was beyond the scope of this study. ${ }^{34}$ Everaert et al. suggested that a coefficient of variation (noise level) of approximately $13 \%$ in the liver region may be clinically acceptable. ${ }^{35}$ Similarly, we found good to excellent image quality (Fig. 5) by using 12\% noise level based on subjective assessment of image quality by independent blinded reviewers.

In this study, we sought to achieve a consistent image noise level over our patient population while maintaining radiation doses as low as reasonably achievable. We assessed image quality based on noise level in the liver, although we recognize that the relationship between noise and image quality is not uniquely determined. ${ }^{36,37}$ A very low $\mathrm{CV}$ could be achieved by applying excessive image smoothing. In this case, liver CV would be low with poor image quality. It would be preferable to evaluate both noise and contrast and aspire to protocols that achieve matched signal to noise or contrast to noise levels for clinically relevant lesion sizes. Evaluating contrast and/or signal levels in patient data is extremely challenging due to the lack of knowledge of true contrast levels and the reality that contrast performance is strongly a function of feature size. While previous work has reported protocols with matched "signal to noise" metrics, their definition of "signal" is not lesion signal that is arguably the clinically relevant metric of interest in oncology imaging. ${ }^{18}$ In fact, our image noise metric, $\mathrm{CV}$, is the inverse of, and therefore provides identical insight as, the SNR metric used in prior work. ${ }^{18}$ Considering lesion signal performance is difficult to evaluate in patient studies, we choose to evaluate performance only over a range of protocols that we have shown in phantom studies to provide reasonable, clinically acceptable signal levels. ${ }^{22}$

Our methodology aimed at minimizing radiological risks to subjects without compromising the diagnostic value of the PET scan. We did so by choosing parameters that best match with PDPs, NECR and CV. The power fits we derived may be used analytically to determine the most appropriate count density for each individual patient. The method produces relations between optimal patient ${ }^{18} \mathrm{~F}$-FDG dose for a given image acquisition protocol and required acquisition time in the

Table IV. Fit of CV with body mass.

\begin{tabular}{|c|c|c|c|c|c|c|c|c|}
\hline \multirow[b]{2}{*}{ Body mass range $(\mathrm{kg})$} & \multicolumn{4}{|c|}{ OSEM3D } & \multicolumn{4}{|c|}{ PSF } \\
\hline & $a$ & $b$ & $R^{2}$ & $p$ value of $t$ test & $a$ & $b$ & $R^{2}$ & $p$ value of $t$ test \\
\hline$<60$ & 1.248 & -0.4085 & 0.79 & 0.0012 & 1.069 & -0.3906 & 0.76 & 0.3847 \\
\hline $60-90$ & 1.172 & -0.3802 & 0.86 & 0.0125 & 1.253 & -0.4102 & 0.90 & 0.0244 \\
\hline$>90$ & 1.129 & -0.3582 & 0.89 & 0.0001 & 1.138 & -0.3821 & 0.90 & 0.0165 \\
\hline
\end{tabular}

Data were fitted with a power adjustment. $a$ and $b$ are fitting parameters [Eq. (5)]. $R^{2}$ : coefficient of determination. $\mathrm{CV}$ : coefficient of variation. OSEM3D: ordered-subsets expectation maximization. PSF: point spread function ordinary Poisson. 
OSEM3D

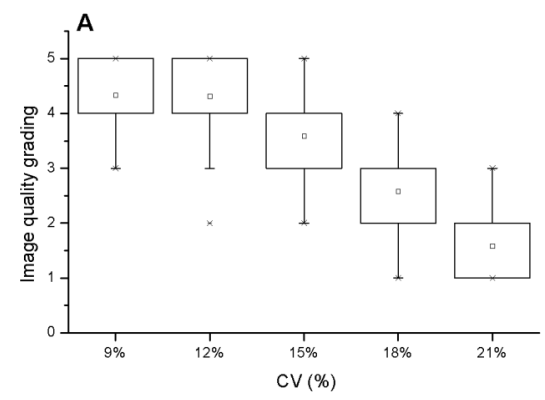

PSF

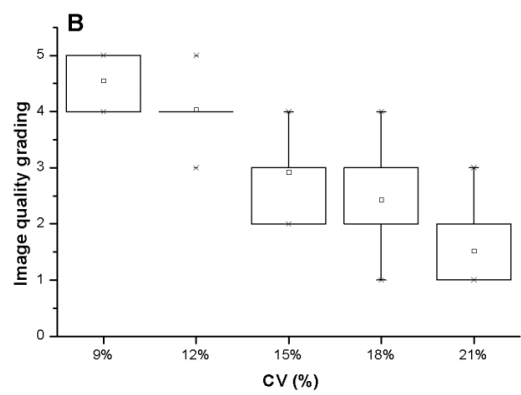

FIG. 5. Image quality by subjective assessment for 90 images (six subjects of each body mass range, five images within $9 \%-21 \%$ noise level per subject) reconstructed with (A) OSEM3D and (B) PSF. The images were graded with a 5-point scale: 1 (poor) to 5 (excellent).

event of a dose bias. For example, an injected activity concentration of $3.7 \mathrm{MBq} / \mathrm{kg}(0.1 \mathrm{mCi} / \mathrm{kg})$ would result in an acquisition time of $2.0 \mathrm{~min} /$ bed for subjects $<60 \mathrm{~kg}, 2.6 \mathrm{~min} /$ bed for subjects $60-90 \mathrm{~kg}$ and $3.4 \mathrm{~min}$ for subjects $>90 \mathrm{~kg}$ to maintain noise levels at approximately $12 \%$ in the OSEM3D reconstruction. With PSF reconstruction, these times decrease to 1.8, 2.0 , and $2.4 \mathrm{~min} / \mathrm{bed}$, respectively. It is important to stress that every institution might decide what noise level is acceptable for its clinical routine. However, by assuring a standardized coefficient variation over a population, we guarantee image quality is maintained.

The results showed that only body mass and BSA remained as significant predictors of $\mathrm{CV}$, after controlling for the other variables (Table III). However, LBM, BMI, and BSA are a function of the body mass, thus having high collinearity, which jeopardizes the multivariate analysis. For this reason, we decided to further explore data regarding body mass in our validation cohort.
The delivery of ${ }^{18} \mathrm{~F}-\mathrm{FDG}$ in Brazil is based on $370 \mathrm{MBq}$ $(10 \mathrm{mCi})$ unique dose for every patient. For this reason, the institution needs to fractionate the dose according to its protocol. Problems concerning logistics also affect ${ }^{18} \mathrm{~F}-\mathrm{FDG}$ availability, imposing some procedures to be performed with limited ${ }^{18} \mathrm{~F}$-FDG activity. Clinical practice is usually affected by delays due to subjects with special needs or due to the need for additional image acquisitions. These issues cause a dispersion in the delays between dose injection and patient scans. The proposed methodology does not address physiological variables arising in case of delay, but it may be applied to correct the bias in image quality by analytically adjusting the scan time per bed position. Other authors also demonstrated that by knowledge of the image quality behavior according to specific patient population, it is possible to find the optimal combination of ${ }^{18}$ F-FDG activity and acquisition time. ${ }^{17,18}$ The application of this methodology showed that image noise improved significantly in clinical practice. The interquartile
A

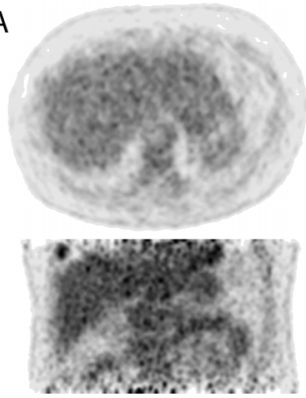

F
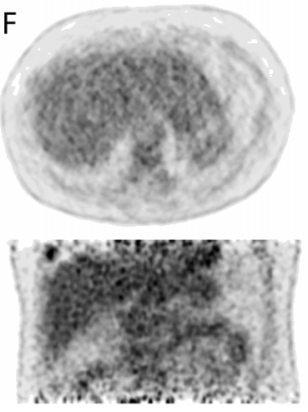

B

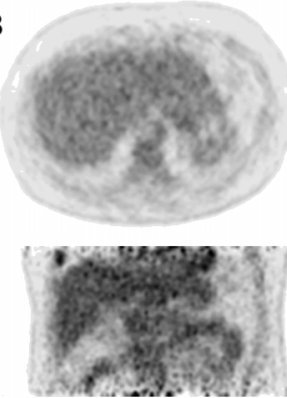

G

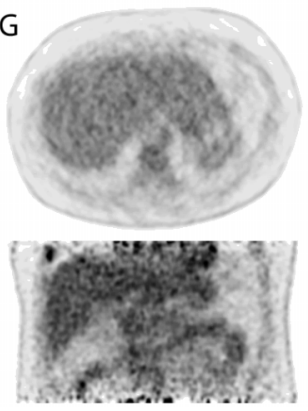

OSEM3D
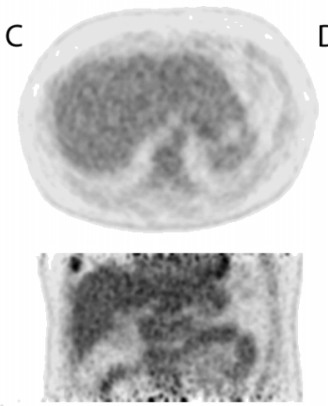

PSF
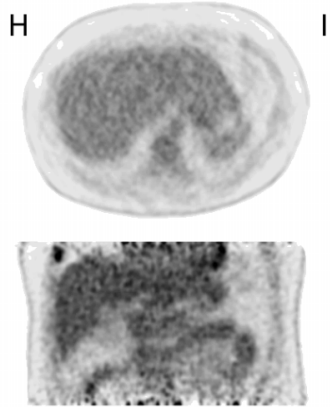
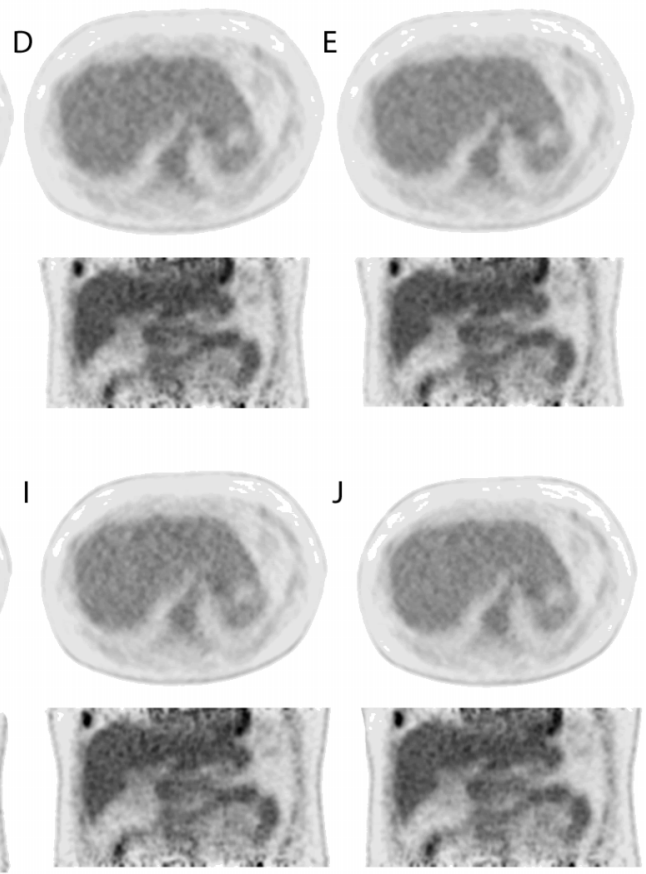

FIG. 6. Coronal and axial slices of a $90 \mathrm{~kg}$ male subject reconstructed with different acquisition time to achieve variable CV: (A) and (F) $21 \%$; (B) and (G) $18 \%$; (C) and (H) $15 \%$; (D) and (I) $12 \%$; (E) and (J) $09 \%$. 
TABLE V. Dose regimen and acquisition parameters adjustments.

\begin{tabular}{lcccccc}
\hline \hline $\begin{array}{l}\text { Recon method } \\
\text { Body mass }(\mathrm{kg})\end{array}$ & $\begin{array}{c}\text { OSEM3D } \\
<60\end{array}$ & $\begin{array}{c}\text { PSF } \\
<60\end{array}$ & $\begin{array}{c}\text { OSEM3D } \\
60-90\end{array}$ & $\begin{array}{c}\text { PSF } \\
60-90\end{array}$ & $\begin{array}{c}\text { OSEM3D } \\
>90\end{array}$ & $\begin{array}{c}\text { PSF } \\
>90\end{array}$ \\
$\begin{array}{l}\text { Dose regimen } \\
(\mathrm{MBq} / \mathrm{kg})\end{array}$ & $\begin{array}{c}\text { T.BED } \\
(\mathrm{min})\end{array}$ & $\begin{array}{c}\text { T.BED } \\
(\mathrm{min})\end{array}$ & $\begin{array}{c}\text { T.BED } \\
(\mathrm{min})\end{array}$ & $\begin{array}{c}\text { T.BED } \\
(\mathrm{min})\end{array}$ & $\begin{array}{c}\text { T.BED } \\
(\mathrm{min})\end{array}$ & $\begin{array}{c}\text { T.BED } \\
(\mathrm{min})\end{array}$ \\
\hline 3.0 & 2.5 & 2.2 & 3.3 & 2.5 & 4.3 & 3.0 \\
3.7 & 2.0 & 1.8 & 2.6 & 2.0 & 3.4 & 2.4 \\
5.2 & 1.5 & 1.3 & 1.9 & 1.4 & 2.5 & 1.7 \\
\hline \hline
\end{tabular}

Note: OSEM3D: ordered-subsets expectation maximization. PSF: point spread function ordinary Poisson. T.BED acquisition time per bed position for a $12 \%$ coefficient of variation [CV, Eq. (5)].

interval in the subjective assessment was bad to good for the first phase of the study, while it was good for the second phase (there was no subject evaluated as bad). It evidences that our methodology provides a predictable result as opposed to the empirical results found in the first phase of the study.

Although PSF reconstruction offers improved performance in terms of partial volume effects and detectability with minimal pixel size, ${ }^{38}$ we chose the same pixel size as in the OSEM3D reconstruction to avoid introducing additional image noise. $^{7}$

Because noise has a different structure in PSF compared to OSEM reconstructions, the values of $\mathrm{CV}$ for different reconstruction methods could not be directly comparable ${ }^{38}$ and the $\mathrm{CV}$ was determined separately for OSEM and PSF, although we found $12 \%$ as a high quality standard for both reconstructions. As we demonstrated, PSF changes the optimal relation between the patient-dependent parameter and the count density. On the other hand, PSF reconstruction overestimates quantitative parameters. ${ }^{39,40}$ Then, we decided not to introduce PSF into clinical routine, despite its higher detectability performance was demonstrated elsewhere. ${ }^{40}$ However, further investigations might demonstrate PSF is a potential tool for protocol optimization since quantitation is harmonized. ${ }^{40}$

A limitation of our study is that it is specific for our population, equipment, and reconstruction. The small number of obese subjects in our population is an additional limitation. A limitation is also analyzing the overall image quality based on the noise level in the liver region, which does not guarantee satisfactory image quality in other regions. However, our methodology allows improvements in image quality and

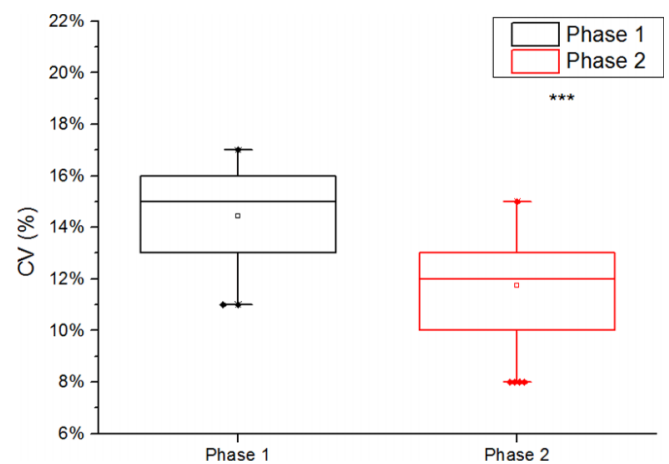

Fig. 7. Image quality based on $\mathrm{CV}$ for the retrospective and prospective subjects, phase $1(n=48)$ and phase $2(n=179)$, respectively. ${ }^{* * *} p<0.0001$, student $t$ test. dosimetry, and we plan to use it or encourage its use for different PET/CT scanners and different populations, including pediatric subjects.

\section{CONCLUSION}

The definition of the optimal ${ }^{18} \mathrm{~F}$-FDG dose regimen and acquisition time for a required image noise level, for different processing and patient-dependent variables in oncological PET/CT scans, and for a group of subjects from the North-East of Brazil was performed. These results may be prospectively used to achieve consistent image quality and optimal radiation doses. Moreover, PSF reconstruction gives rise to alternative protocols. Optimized use of ${ }^{18} \mathrm{~F}-\mathrm{FDG}$, prevention of image artifacts, and high quality performance will assist a costeffective operation of PET/CT centers.

\section{ACKNOWLEDGMENTS}

The authors thank the Comissão Nacional de Energia Nuclear (CNEN, Brazil) and the International Atomic Energy Agency (IAEA) for the efforts to develop the nuclear medicine at their institution through a Technical Cooperation project. The authors also take this opportunity to thank Professor Adam M. Alessio for the constructive comments to improve the paper. No potential conflict of interest relevant to this paper is reported. All data collected and presented in this paper were derived from human patients, after signing official consent and participation forms.

\footnotetext{
a) Author to whom correspondence should be addressed. Electronic mail: vinicius@radtec.com.br; Telephone: +55 71 3281-6891; Fax. +55 71 3281-6327.

${ }^{1}$ IAEA-TECDOC, 1340, ISSN: 1011-4289, (ㅇ IAEA, Austria, Vienna, IAEA, 71, 2003.

${ }^{2}$ E. M. Rohren, T. G. Turkington, and R. E. Coleman, "Clinical applications of PET in oncology 1," Radiology 231, 305-332 (2004).

${ }^{3}$ P. E. Valk, Positron Emission Tomography: Basic Sciences (Springer Science \& Business Media, London, 2003).

${ }^{4}$ M. MacManus, U. Nestle, K. E. Rosenzweig, I. Carrio, C. Messa, O. Belohlavek, M. Danna, T. Inoue, E. Deniaud-Alexandre, and S. Schipani, "Use of PET and PET/CT for radiation therapy planning: IAEA expert report 2006-2007,’ Radiother. Oncol. 91, 85-94 (2009).

${ }^{5}$ ACR-SPR practice parameter for performing FDG-PET/CT in oncology, 2014.

${ }^{6}$ R. Boellaard, M. J. O'Doherty, W. A. Weber, F. M. Mottaghy, M. N. Lonsdale, S. G. Stroobants, W. J. Oyen, J. Kotzerke, O. S. Hoekstra, and
} 
J. Pruim, "FDG PET and PET/CT: EANM procedure guidelines for tumour PET imaging: Version 1.0,' Eur. J. Nucl. Med. Mol. Imaging 37, 181-200 (2010).

${ }^{7}$ R. Boellaard, R. Delgado-Bolton, W. J. Oyen, F. Giammarile, K. Tatsch, W. Eschner, F. J. Verzijlbergen, S. F. Barrington, L. C. Pike, and W. A. Weber, "FDG PET/CT: EANM procedure guidelines for tumour imaging: Version 2.0,’ Eur. J. Nucl. Med. Mol. Imaging 42, 328-354 (2015).

${ }^{8}$ B. Krause, T. Beyer, A. Bockisch, D. Delbeke, J. Kotzerke, V. Minkov, M. Reiser, and N. Willich, "FDG-PET/CT in oncology," Nuklearmedizin 46, 291-301 (2007).

${ }^{9}$ H. Fukukita, M. Senda, T. Terauchi, K. Suzuki, H. Daisaki, K. Matsumoto, Y. Ikari, and M. Hayashi, "Japanese guideline for the oncology FDG-PET/CT data acquisition protocol: Synopsis of version 1.0," Ann. Nucl. Med. 24, 325-334 (2010).

${ }^{10}$ D. Delbeke, R. E. Coleman, M. J. Guiberteau, M. L. Brown, H. D. Royal, B. A. Siegel, D. W. Townsend, L. L. Berland, J. A. Parker, K. Hubner, M. G. Stabin, G. Zubal, M. Kachelriess, V. Cronin, and S. Holbrook, "Procedure guideline for tumor imaging with ${ }^{18}$ F-FDG PET/CT 1.0,” J. Nucl. Med. 47, 885-895 (2006).

${ }^{11}$ Y. Masuda, C. Kondo, Y. Matsuo, M. Uetani, and K. Kusakabe, "Comparison of imaging protocols for ${ }^{18} \mathrm{~F}-\mathrm{FDG}$ PET/CT in overweight patients: Optimizing scan duration versus administered dose," J. Nucl. Med. 50, 844-848 (2009).

${ }^{12}$ T. Chang, G. Chang, S. Kohlmyer, J. W. Clark, Jr., E. Rohren, and O. R. Mawlawi, "Effects of injected dose, BMI and scanner type on NECR and image noise in PET imaging," Phys. Med. Biol. 56, 5275-5285 (2011).

${ }^{13}$ G. Akamatsu, K. Ishikawa, K. Mitsumoto, T. Taniguchi, N. Ohya, S. Baba, K. Abe, and M. Sasaki, "Improvement in PET/CT image quality with a combination of point-spread function and time-of-flight in relation to reconstruction parameters," J. Nucl. Med. 53, 1716-1722 (2012).

${ }^{14}$ A. Nagaki, M. Onoguchi, and N. Matsutomo, "Patient weight-based acquisition protocols to optimize ${ }^{18}$ F-FDG PET/CT image quality,” J. Nucl. Med. Technol. 39, 72-76 (2011).

${ }^{15}$ A. M. Alessio, P. E. Kinahan, V. Manchanda, V. Ghioni, L. Aldape, and M. T. Parisi, "Weight-based, low-dose pediatric whole-body PET/CT protocols," J. Nucl. Med. 50, 1570-1578 (2009).

${ }^{16}$ F. Molina-Duran, D. Dinter, F. Schoenahl, S. O. Schoenberg, and G. Glatting, "Dependence of image quality on acquisition time for the PET/CT biograph mCT," Z. Med. Phys. 24, 73-79 (2014).

${ }^{17}$ M. Namías, A. R. Osorio, G. L. Bruno, C. González, Y. Blumen-Krantz, and P. E. Parma, "Optimización del tiempo de adquisición en PET/CT de cuerpo entero con ${ }^{18}$ F-FDG," Rev. Asociación Argentina Biol. Med. Nucl. 2, 13-16 (2012).

${ }^{18}$ E. H. de Groot, N. Post, R. Boellaard, N. R. Wagenaar, A. T. Willemsen, and J. A. van Dalen, "Optimized dose regimen for whole-body FDG-PET imaging,” EJNMMI Res. 3, 63-73 (2013).

${ }^{19}$ C. C. Watson, M. E. Casey, B. Bendriem, J. P. Carney, D. W. Townsend, S. Eberl, S. Meikle, and F. P. DiFilippo, "Optimizing injected dose in clinical PET by accurately modeling the counting-rate response functions specific to individual patient scans," J. Nucl. Med. 46, 1825-1834 (2005).

${ }^{20}$ B. W. Jakoby, Y. Bercier, C. C. Watson, B. Bendriem, and D. W. Townsend, "Performance characteristics of a new LSO PET/CT scanner with extended axial field-of-view and PSF reconstruction," IEEE Trans. Nucl. Sci. 56, 633-639 (2009).

${ }^{21}$ C. C. Queiroz, V. O. Menezes, M. A. D. Machado, M. Namias, A. G. Santos, D. A. Queijo, L. O. Vieira, L. J. L. Sampaio, and A. O. Vigário, "Avaliação das características de desempenho de um PET/CT LSO com campo de visão axial extendido," in XXVI Congresso Brasileiro de Medicina Nuclear (SBMN, Salvador, 2012).

${ }^{22}$ N. Paquet, A. Albert, J. Foidart, and R. Hustinx, "Within-patient variability of ${ }^{18}$ F-FDG: Standardized uptake values in normal tissues," J. Nucl. Med. 45, 784-788 (2004).
${ }^{23}$ S. Strother, M. Casey, and E. Hoffman, "Measuring PET scanner sensitivity: Relating countrates to image signal-to-noise ratios using noise equivalents counts,” IEEE Trans. Nucl. Sci. 37, 783-788 (1990).

${ }^{24}$ I. S. Armstrong, M. D. Kelly, H. A. Williams, and J. C. Matthews, "Impact of point spread function modelling and time-of-flight on FDG uptake measurements in lung lesions using alternative filtering strategies," EJNMMI Phys. 1, 99-116 (2014).

${ }^{25}$ R. Boellaard, W. J. Oyen, C. J. Hoekstra, O. S. Hoekstra, E. P. Visser, A. T. Willemsen, B. Arends, F. J. Verzijlbergen, J. Zijlstra, and A. M. Paans, "The Netherlands protocol for standardisation and quantification of FDG whole body PET studies in multi-centre trials," Eur. J. Nucl. Med. Mol. Imaging 35, 2320-2333 (2008).

${ }^{26}$ R. L. Wahl, H. Jacene, Y. Kasamon, and M. A. Lodge, "From RECIST to PERCIST: Evolving considerations for PET response criteria in solid tumors," J. Nucl. Med. 50, 122S-150S (2009).

${ }^{27}$ A. Keys, F. Fidanza, M. J. Karvonen, N. Kimura, and H. L. Taylor, "Indices of relative weight and obesity," J. Chronic Dis. 25, 329-343 (1972).

${ }^{28}$ R. Hume, "Prediction of lean body mass from height and weight," J. Clin. Pathol. 19, 389-391 (1966).

${ }^{29}$ D. Du Bois and E. Du Bois, "A formula to estimate the approximate surface area if height and weight be known. 1916," in Nutrition (Nutrition, Burbank, Los Angeles County, CA, 1989), Vol. 5, p. 303.

${ }^{30}$ S. L. Brady and R. A. Kaufman, "Investigation of American Association of Physicists in Medicine Report 204 size-specific dose estimates for pediatric CT implementation," Radiology 265, 832-840 (2012).

${ }^{31}$ J. A. Christner, N. N. Braun, M. C. Jacobsen, R. E. Carter, J. M. Kofler, and C. H. McCollough, "Size-specific dose estimates for adult patients at CT of the torso," Radiology 265, 841-847 (2012).

${ }^{32}$ G. El Fakhri, P. A. Santos, R. D. Badawi, C. H. Holdsworth, A. D. Van Den Abbeele, and M. F. Kijewski, "Impact of acquisition geometry, image processing, and patient size on lesion detection in whole-body ${ }^{18} \mathrm{~F}$-FDG PET," J. Nucl. Med. 48, 1951-1960 (2007).

${ }^{33}$ A. M. Alessio, M. Sammer, G. S. Phillips, V. Manchanda, B. C. Mohr, and M. T. Parisi, "Evaluation of optimal acquisition duration or injected activity for pediatric ${ }^{18}$ F-FDG PET/CT,' J. Nucl. Med. 52, 1028-1034 (2011).

${ }^{34}$ T. Bach-Gansmo, J. Dybvik, T. Adamsen, and A. Naum, "Variation in urinary excretion of FDG, yet another uncertainty in quantitative PET," Acta Radiol. Short Rep. 1, 26-28 (2012).

${ }^{35}$ H. Everaert, C. Vanhove, T. Lahoutte, K. Muylle, V. Caveliers, A. Bossuyt, and P. R. Franken, "Optimal dose of ${ }^{18}$ F-FDG required for whole-body PET using an LSO PET camera,’ Eur. J. Nucl. Med. Mol. Imaging 30, 1615-1619 (2003).

${ }^{36}$ C. Riddell, R. E. Carson, J. A. Carrasquillo, S. K. Libutti, D. N. Danforth, M. Whatley, and S. L. Bacharach, "Noise reduction in oncology FDG PET images by iterative reconstruction: A quantitative assessment," J. Nucl. Med. 42, 1316-1323 (2001).

${ }^{37}$ C. Schmidtlein, B. Beattie, D. Bailey, T. Akhurst, W. Wang, M. Gönen, A. Kirov, and J. Humm, "Using an external gating signal to estimate noise in PET with an emphasis on tracer avid tumors," Phys. Med. Biol. 55, 6299-6326 (2010).

${ }^{38}$ S. Tong, A. Alessio, and P. Kinahan, "Noise and signal properties in PSFbased fully 3D PET image reconstruction: An experimental evaluation," Phys. Med. Biol. 55, 1453-1473 (2010).

${ }^{39}$ G. Akamatsu, K. Mitsumoto, T. Taniguchi, Y. Tsutsui, S. Baba, and M. Sasaki, "Influences of point-spread function and time-of-flight reconstructions on standardized uptake value of lymph node metastases in FDG-PET,' Eur. J. Radiol. 83(1), 226-230 (2014).

${ }^{40}$ F. L. Andersen, T. L. Klausen, A. Loft, T. Beyer, and S. Holm, "Clinical evaluation of PET image reconstruction using a spatial resolution model,' Eur. J. Radiol. 82(5), 862-869 (2013). 